\title{
Erratum to the Entry: Gut Mucosal Atrophy
}

Indraneil Mukherjee ${ }^{1}$ and Khanjan H. Nagarsheth ${ }^{2}$

${ }^{1}$ The Southeastern Center for Digestive Disorders \& Pancreatic Cancer, Advanced Minimally Invasive \& Robotic Surgery, Florida Hospital Tampa, Tampa, FL, USA

${ }^{2} \mathrm{R}$ Adams Cowley Shock Trauma Center, University of Maryland School of Medicine, Baltimore, MD, USA

Owing to an unfortunate oversight the first published version of this entry contained an error regarding the authorship which has now been corrected. 